For publication as Chapter 2 of "New Science of Learning: Cognition, Computers and Collaboration in Education.” Edited by Myint Swe Khine and Issa M. Saleh.

\title{
GROUP COGNITION AS A FoUndATION FOR THE NEW SCIENCE OF LEARNING Gerry Stahl
}

\author{
Gerry@GerryStahl.net \\ College of Information Science \& Technology \\ Drexel University, Philadelphia, PA 19104 USA
}

\begin{abstract}
In the new global world, it is time to recognize that knowledge is primarily produced socially and progressively, not just by spontaneous acts of isolated minds. Individuals participate in this as knowledge learners, knowledge users and knowledge builders, predominantly through their interactions in small groups. The cognitive work of small groups can be distinct from individual thinking and community knowledge processing.

We need a new science of group learning to complement our sciences of individual learning and community knowledge building. In particular, we need a science that will help us to realize the potential of computer networking to foster the formation of virtual groups and computersupported collaborative learning.

The construct of group cognition provides a theoretical and practical foundation for developing the needed science, for analyzing the work of small groups and for designing effective collaboration software. The Virtual Math Teams Project provides a model of such scientific research. Along with related explorations of group processes, it has already started to produce concrete analyses of group cognition and to develop a multi-faceted online educational environment.

A science of group cognition can systematically provide findings that are objective, reliable and generalizable through its interpretive case studies. With its focus on the group as the unit of description, group cognition joins other post-cognitive theories, which extend the analysis of cognition beyond the psychological individual. As the pivotal middle ground in which knowledge is primordially co-constructed, the group provides a foundation for sciences of learning at the individual, small group and community levels.
\end{abstract}

Keywords: Group cognition, Collaborative learning, CSCL, Distributed cognition, Small groups.

"It takes a village to raise a child." This ancient African proverb reflects the direct bearing of social relations on learning. In pre-industrial society, the individual, family-of-origin, extended family, clan, tribe, village and culture blended into one another almost seamlessly. With the rise of capitalism, the individual was uprooted from its social ground and celebrated as a free spirit- 
in order to compete unencumbered on the labor market (Marx, 1867/1976). With globalization, the forces of production require information-processing tasks that exceed the capabilities of individual minds, necessitating the formation of well-coordinated knowledge-building teams. Thus, Hillary Clinton's use of the proverb (Clinton, 1996) not only looks back nostalgically to a romanticized past of homogeneous villages and neighborly towns, but also reflects the realities of our increasingly interconnected global village.

The nature of learning is transformed-along with other aspects of human social existence - by societal upheavals. But our thinking about learning lags behind these changes. Furthermore, the evolution of social institutions is uneven, and past forms linger on in confusing mixtures. So our theories of learning, founded upon popular conceptions or "folk theories" (Bereiter, 2002), confuse individual, group and community characteristics, while still exalting the individual learner.

It is time for a new science of learning because, as Bob Dylan already announced to the youth social movement of the 1960s, "the times they are a-changin'." Foremost in our reconceptualization of learning must be a recognition not only of the role of the (post-modern) village, but also of the often ephemeral small groups that mediate between the tangible individual learner and the insubstantial communities within which the learner comes to participate. Imagine the gatherings of friends who listened to Dylan's lyrics together, forming cadre of the new age awakening around the world a half century ago. The interactions in these peer groups contributed to the new identities of the individuals involved as well as of their generation. Creative ways of thinking, making meaning and viewing the world emerged. The scientific disciplines with their traditional methods are not equipped to analyze the interpenetration of such learning processes at the individual, small-group and community levels.

\section{The Need for a New Science of Group Cognition}

The idea of a science of group cognition was originally motivated by issues of software design for collaborative learning. The design of software to support group work, knowledge building and problem solving should be built on the foundation of an understanding of the nature of group interaction and group meaning making. However, previous research in computer-supported collaborative learning (CSCL) is mostly based on an ad hoc collection of incommensurable theories, which are not grounded in an explicit investigation of group interaction. What is needed is a science of group interaction focused on the group level of description to complement psychological theories of individuals and social theories of communities.

CSCL is fundamentally different from other domains of study in the learning sciences (Stahl, 2002). It takes as its subject matter collaborative learning, that is, what takes place when small groups of workers or students engage together in cognitive activities like problem solving or knowledge building (Koschmann, 1996; Stahl, 2006, ch. 11). On a theoretical level, CSCL is strongly oriented toward Vygotsky (1930/1978), who stressed that learning and other higher psychological processes originally take place socially, intersubjectively. Piaget (1985), too, pointed to inter-subject processes like conflicting perspectives as a fundamental driver for creativity and cognitive development. Despite this powerful insight, even Vygotsky, Piaget and their followers generally maintain a psychological focus on the individual mind in their empirical 
studies and do not systematically investigate the intersubjective phenomena of small-group interaction.

A science of group interaction would aim to unpack what happens at the small-group unit of analysis (Stahl, 2004b). Thus, it would be particularly relevant for CSCL, but may not be as directly applicable to other forms of learning, where the individual or the community level predominates. As a science of the group, it would complement existing theories of acting, learning and cognition, to the extent that they focus either on the individual or the community or that they reduce group phenomena to these other levels of description.

In the chapters of Studying Virtual Math Teams (Stahl, 2009) and of Group Cognition (Stahl, 2006), my colleagues and I have reviewed some of the research literature on small-group learning, on small-group processes and on collaborative mathematics. We have noticed that small-group studies generally look for quantitative correlations among variables - such as the effect of group size on measures of participation-rather than trying to observe group knowledge-building processes. Studies of small-group processes from psychology, sociology and other social sciences also tend to focus on non-cognitive aspects of group process or else attribute all cognition to the individual minds rather than to group processes. This was true of writings on cooperative learning in the 1970s and 1980s as well, e.g., Johnson \& Johnson (1989).

There are some notable exceptions; in particular, we viewed (Barron, 2000; 2003; Cohen et al., 2002; Sawyer, 2003; Schwartz, 1995) as important preliminary studies of group cognition within the learning sciences. However, even theories in cognate fields that seem quite relevant to our concerns, like distributed cognition (Hutchins, 1996), actor-network theory (Latour, 2007), situated cognition (Lave \& Wenger, 1991), ethnomethodology (Garfinkel, 1967) and activity theory (Engeström, 1987) adopt a different focus, generally on interaction of individuals with artifacts rather than among people, indicating an orientation to the larger community scale of social sciences.

Recent commentaries on situated cognition (Robbins \& Aydede, 2009) and distributed cognition (Adams \& Aizawa, 2008) frame the issues at the individual level, even reducing all cognitive phenomena to neural phenomena. At the other extreme, social theories focus on community phenomena like division of labor, apprenticeship training, linguistic structure, laboratory organization. For all its insight into small-group interaction and its analysis, even ethnomethodology maintains a sociological perspective, concerned with linguistic communities. Similarly, even when activity theory addresses the study of teams - in the most detail in Chapter 6 of (Engeström, 2008) - it is mostly concerned with the group's situation in the larger industrial and historic context; rather than analyzing how groups interactionally build knowledge it paraphrases how they deal politically with organizational management issues. These theories provide valuable insights into group interaction, but none of them thematizes the small-group level as a domain of scientific study. As sciences, these are sciences of the individual or of the society, not of the collaborative group.

Each of the three levels of description is populated with a different set of phenomena and processes. For instance, individuals in a chat or threaded discussion interpret recent postings and design new postings in response, the group constructs, maintains and repairs a joint problem space and the community evolves its practices and institutions of social organization. The description of the individual level is the province of psychology; that of the community is the realm of sociology or anthropology; the small-group level has no corresponding science. 
A science of group interaction would take its irreducible position between the psychological sciences of the individual and the social sciences of the community-much as biology analyzes phenomena that are influenced by both chemicals and organisms without being reducible to either. The science of group interaction would fill a lacuna in the multi-disciplinary work of the human sciences - including the learning sciences. This science would not be primarily oriented toward the "low level" processes of groups, such as mechanical or rote behaviors, but would be concerned with the accomplishment of creative intellectual tasks. Intellectual teamwork, knowledge work and knowledge-building activities would be prototypical objects of study. The focus would be on group cognition.

The bifurcation of the human sciences into individual and societal creates an irreconcilable opposition between individual creative freedom and restrictive social institutions. A science of group cognition would flesh out the concept of structuration, demonstrating with detailed analyses of empirical data how group interactions can mediate between individual behavior and social practices (Stahl, 2009, ch. 11).

\section{The Construct of Group Cognition}

The term group cognition does not signify an object or phenomenon to analyze like brain functions or social institutions (Stahl, 2004a). It is a proposal for a new science or focus within the human sciences. It hypothesizes:

When small groups engage in cooperative problem solving or collaborative knowledge building, there are distinctive processes of interest at the individual, small-group and community levels of analysis, which interact strongly with each other. The science of group cognition is the study of the processes at the small-group level.

The science of group cognition is a human science, not a predictive science like chemistry, nor a predominantly quantitative one like physics. It deals with human meanings in unique situations, necessarily relying upon interpretive case studies and descriptions of inter-personal processes.

Processes at the small-group level are not necessarily reducible to processes of individual minds, nor do they imply the existence of some sort of group mind. Rather, they may take place through the weaving of semantic and indexical references within a group discourse. The indexical field (Hanks, 1992) or joint problem space (Teasley \& Roschelle, 1993) co-constructed through the sequential interaction of a group (Çakır, Zemel \& Stahl, 2009) has the requisite complexity to constitute an irreducible cognitive act in its own right. The rise of cognitive science in the 1980s broadened the definition of "cognition" beyond an activity of human minds in order to include the artificial intelligence of computers. What counts as cognitive is now a matter of computational complexity. Anything that can compute well enough to play chess or prove theorems can be a cognitive agent — whether it is a person, computer or collaborative small group (Stahl, 2005).

Largely because of its linguistic form, the noun phrase "group cognition" is often taken to refer to some kind of physical or mental object. But it is a theoretical construct, not an object, as indicated by the hypothesis stated above. Commonsensical folk theories assume that we generally talk about physical objects. However, if one looks closely, most sciences deal with hypothesized entities, not physical objects; mental representations are a prime example at the 
individual level and cultural norms or social rules at the community level. Mental entities cannot be physically located - even with the latest MRI machines - and neither can rules.

The group that engages in group cognition is not necessarily a set of physical people who interact together in the present moment. For example, group processes of problem solving, meaning making and knowledge building can be revealed in computer logs of chat or threaded discussion, where the people who contributed are now long gone. The interaction is captured and remains accessible through the log. The "interaction" of textual artifacts is not like physical interactions (the cause-and-effect interactions of instantaneously colliding billiard balls in physics or the face-to-face embodied interactions of people in the present moment), but can bring together references from the distant past or into the future (the mediator interactions of a network of actors). As Latour notes, "a banal conversation may become a terribly complex chain of mediators where passions, opinions, and attitudes bifurcate at every turn" (2007, p. 39). The textual interaction itself constitutes the discourse as a group interaction, by, for instance, addressing proposals to the group as a whole (Lerner, 1993) and referencing among postings in complex ways. Of course, the subtle textual interactions were originally designed by the people situated in the discourse and typing the postings, but the persistent interaction takes place through the textual medium by means of the meditational agency of the textual elements (Zemel \& Çakir, 2007).

Rather than taking the number of human bodies as a definition of "small group," one could consider the complexity of the interaction among postings from different people. Productive knowledge building, creative meaning making and innovative group cognition take place through dense networks of referencing, in which the postings of each participant takes into account the past postings and the potential future responses of all the other participants. This contrasts not only with a case in which someone's postings only refers to his own other postings (individual cognition), but also with cases where there are clusters of mutual referencing connected by sparse referencings (community cognition). The group cognition case involves active generation of shared understandings, whereas the community case requires the mediation over time of persistent representations, artifacts and dissemination processes in order to cross the sparse connections.

Not only is it wrong to associate the construct of the "group" in a simple way with a set of people, it is wrong to think of it in terms of a type of physical object or assemblage distinguished from other types. The distinction between individual, group and community might better be conceptualized by developing Vygotsky's (1930/1978) sketchy and problematic concepts of "internalization" and "externalization." Vygotsky argued that the higher psychological processes of humans (i.e., cognition) developed through interaction between people. These processes could subsequently (through complicated, extended processes of mediation that are not well understood) be "internalized" as unvocalized self-talk and mental skills, and externalized as artifacts and other elements of a community's culture. Taking this approach, the theory of group cognition is interested in exploring the pivotal meaning-making processes of group interaction, which can subsequently lead to both individual thought and community norms. Such a view differentiates individual, group and community levels as functionally distinct. It lends a foundational role to group cognition within a new science of learning. 


\section{ThE GROUP UNIT OF DESCRIPTION}

The theory of group cognition stakes out a new domain for exploration: the domain of group meaning-making processes. Importantly, it distinguishes this domain from the traditional domains of sciences of individual learning and of the development of social practices in communities. Virtually all discussions in the learning sciences have been ambiguous in their terminology when it comes to distinguishing the individual, group and cultural levels of description. My own writings have used the relevant terminology in a loose way. Therefore, it may be helpful to try to codify a set of terms for speaking at the three different levels (see Table 1).

Table 1. Terminology distinguishing the three levels of description.

\begin{tabular}{|c|c|c|c|}
\hline Level of description & Individual & Group & Community \\
\hline Role & Person / student & Group participant & Community member \\
\hline Adjective & Personal & Collaborative & Social \\
\hline Object of analysis & Mind & Discourse & Culture \\
\hline Unit of analysis & $\begin{array}{l}\text { Mental } \\
\text { representation }\end{array}$ & $\begin{array}{l}\text { Utterance response } \\
\text { pair }\end{array}$ & Mediating artifact \\
\hline Form of knowledge & Subjective & Intersubjective & Cultural \\
\hline Form of meaning & Interpretation & $\begin{array}{l}\text { Shared understanding, } \\
\text { joint meaning making, } \\
\text { common ground }\end{array}$ & $\begin{array}{l}\text { Domain vocabulary, } \\
\text { artifacts, institutions, } \\
\text { norms, rules }\end{array}$ \\
\hline Learning activity & Learn & Build knowledge & Science \\
\hline $\begin{array}{l}\text { Way to accomplish } \\
\text { cognitive tasks }\end{array}$ & Skill, behavior & Group method & $\begin{array}{l}\text { Member method I } \\
\text { social practice }\end{array}$ \\
\hline Communication & Thought & Interaction & Membership \\
\hline Mode of construction & Constructed & Co-constructed & Socially constructed \\
\hline $\begin{array}{l}\text { Context of cognitive } \\
\text { task }\end{array}$ & Personal problem & Joint problem space & Problem domain \\
\hline Context of activity & Embodiment & Situation & World \\
\hline Referential system & Associations & Indexical field & Cultural world \\
\hline Form of existence & Being there & Being with & Folk \\
\hline Temporal structure & $\begin{array}{l}\text { Subjective } \\
\text { experiential internal } \\
\text { time }\end{array}$ & $\begin{array}{l}\text { Co-constructed shared } \\
\text { temporality }\end{array}$ & $\begin{array}{l}\text { Measurable objective } \\
\text { time }\end{array}$ \\
\hline Theory of cognition & Constructivist & Post-cognitive & Socio-cultural \\
\hline Science & $\begin{array}{l}\text { Cognitive and } \\
\text { educational } \\
\text { psychology }\end{array}$ & Group cognition & $\begin{array}{l}\text { Sociology, } \\
\text { anthropology, } \\
\text { linguistics }\end{array}$ \\
\hline
\end{tabular}

Of course, some of this classification of terms is arbitrary and inconsistent with prior usage. In particular, the terms related to groups and cultures have not been kept distinct. Even Vygotsky, who pioneered in distinguishing the social from the individual, would use terms like "social" and "intersubjective" to apply to anything from a dyad to all of society. Within the learning sciences, "knowledge building" has been used at every level, resulting in controversy about whether classrooms are communities-of-practice, for instance. The characteristics of scientific research communities were projected onto classrooms, project groups and individuals 
without carefully distinguishing their different ways of building knowledge. Table 1 therefore suggests, for instance, using "intersubjective" at the group level and "social" at the community level. It suggests using "joint," "shared," "collaborative" and "co-constructed for group phenomena.

Such ambiguity of terminological usage even led to pseudo-problems, which can now be resolved by the theory of group cognition, showing how small groups mediate between the individual and the community phenomena. To take one example, the seeming irreconcilability of subjective and objective time can be bridged by considering how small groups co-construct their shared temporal reference system. Significantly, the co-construction can be observed in logs of interaction and analyzed in detail-which cannot be done for either the subjective sense of internal time (Husserl, 1917/1991) or the abstract dimension of scientifically measured time (Heidegger, 1927/1996).

The move from the individual to the group level of description as foundational entails an important philosophical step: from cognitivism to post-cognitivism. This step has its basis in philosophy (Hegel, 1807/1967; Heidegger, 1927/1996; Marx, 1867/1976; Merleau-Ponty, 1945/2002; Wittgenstein, 1953), in social science (Bourdieu, 1972/1995; Geertz, 1973; Giddens, 1984a) and in analytic methods of ethnomethodology and conversation analysis (Garfinkel, 1967; Livingston, 1987; Sacks, 1962/1995; Schegloff, 2007). Post-cognitive theories influential in CSCL and the learning sciences include: the critique of cognitivism (Dreyfus, 1972; Polanyi, 1962; Schön, 1983; Winograd \& Flores, 1986), situated action (Suchman, 1987), situated learning (Lave \& Wenger, 1991), activity theory (Engeström, 1987), distributed cognition (Hutchins, 1996), actor-network theory (Latour, 2007) and knowledge building (Scardamalia \& Bereiter, 1996).

In two seminal statements of post-cognitivist theory, Hutchins has explicitly pointed to group cognitive phenomena: "Cognitive processes may be distributed across the members of a social group" (Hollan, Hutchins \& Kirsh, 2000, p. 176). "The cognitive properties of groups are produced by interaction between structures internal to individuals and structures external to individuals" (Hutchins, 1996, p. 262). "The group performing the cognitive task may have cognitive properties that differ from the cognitive properties of any individual" (Hutchins, 1996, p. 176). However, rather than focusing on these group phenomena in detail, he analyzes sociotechnical systems and the cognitive role of highly developed artifacts (airplane cockpits, ship navigation tools). Certainly, these artifacts have encapsulated past cultural knowledge (community cognition), and Hutchins' discussions of this are important. But in focusing on what is really the community level - characteristically for a cultural anthropologist-he does not analyze the cognitive meaning making of the group itself (the active navigational team).

In general, the related literature on small groups and on post-cognitivist phenomena provide some nice studies of the pivotal role of small groups, but do not account for this level of description theoretically. They are almost always in the final analysis based on either a psychological view of individuals or a sociological view of rules, etc. at the community level. None of them have a foundational conception of small groups as a distinct level. They confuse talk at the group level and at the social level, and they lack a developed account of the relationships between individual, group and community.

If we take group phenomena seriously as "first-class objects" of our theory, then we can study: interpersonal trains of thought, shared understandings of diagrams, joint problem 
conceptualizations, common references, coordination of problem-solving efforts, planning, deducing, designing, describing, problem solving, explaining, defining, generalizing, representing, remembering and reflecting as a group. In our studies, we will see the groupcognitive accomplishments emerge from the network of meaningful references built up by, for instance, textual postings in online chat. We will see how the group and its cognitive accomplishments are enacted in situated interaction.

\section{A Model of the NeW SCience}

Having motivated the development of a science of group cognition as future work, let us see how the Virtual Math Teams (VMT) Project (Stahl, 2009) may have begun to prepare the way. Preparing for a new science requires three major undertakings:

(a) The domain of the science must not only be defined, it must be explored and captured in the form of a data corpus.

(b) Methods for analyzing the data must be selected, adapted, refined and mastered.

(c) Analytic findings must be organized in terms of a framework of theoretical conceptualizations.

The Virtual Math Teams Project at Drexel University has approached these tasks by:

(a) Creating a synchronous online service in which small groups of students engaged in problem-solving work in mathematics,

(b) Conducting chat interaction analysis of a number of case studies from the data recorded in that service and

(c) Conceptualizing some of the features of the small-group interactions that were observed.

The first step in the VMT design-based research process was to start simply and see what issues came up. We had seen in face-to-face case studies that there were problems with (i) recording and transcribing the verbal interaction, (ii) capturing the visual interaction and (iii) knowing about all the influences on the interaction. We decided to form groups of students who did not know each other and who only interacted through text chat. Students were recruited through the Math Forum at Drexel University, an established online resource center. We used AIM, AOL's Instant Messaging system, which was freely available and was already familiar to many students. We included a researcher in the chat room with each small group of students. The facilitator told the students their math task, dealt with any technical difficulties, posted drawings from the students on a web page where they could be seen by all the students, notified the group when the session was over and saved an automatically generated log of the chat. In this way, we obtained a complete and objective log of the interaction, captured everything that the students shared on their computers and excluded any unknown influences from affecting the interaction.

The issue of including everything affecting the interaction is a subtle issue. Of course, the interaction is influenced by the life histories, personalities, previous knowledge and physical environment of each student. A student may have windows other than AIM open on the computer, including Internet browsers with math resources. A student may be working out math problems on a piece of paper next to the computer. Also, a student may leave the computer for some time to eat, listen to music, talk on the phone, and so on without telling anyone in the chat. 
In such ways, we do not have information about everything involved in a particular student's online experience. We do not even know the student's gender or age. We do not know if the student is shy or attractive, speaks with an accent or stutters. We do not know if the student usually gets good grades or likes math. We do not know what the student is thinking or feeling. We only know that the students are in an approximate age group and academic level-because we recruited them through teachers. However, the VMT Project is only concerned with analyzing the interaction at the group unit of analysis. Notice that the things that are unknown to us as researchers are also unknown to the student group as a whole. The students do not know specifics about each other's background or activities - except to the extent that these specifics are brought into the chat. If they are mentioned or referenced in the chat, then we can be aware of them to the same extent as are the other students.

The desire to generate a complete record for analysis of everything that was involved in a team's interaction often conflicted with the exploration of technology and service design options. For instance, we avoided speech-based interaction (VOIP, Skype, WIMBA) and support for individual work (e.g., whiteboards for individual students to sketch ideas privately), because these would complicate our review of the interactions. We tried to form teams that did not include people who knew each other or who could interact outside of the VMT environment.

In addition to personal influences, the chat is responsive to linguistic and cultural matters. Of course, both students and researchers must know English to understand the chats. In particular, forms of English that have evolved with text chat and cell-phone texting have introduced abbreviations, symbols and emoticons into the online language. The linguistic subculture of teenagers also shows up in the VMT chats. An interdisciplinary team of researchers comes in handy for interpreting the chats. In our case, the research team brought in experience with online youth lingo based on their backgrounds as Math Forum staff, teachers or parents.

The early AIM chats used simple math problems, taken from standardized math tests and Math Forum Problems-of-the-Week. One experiment to compare individual and group work used problems from a standardized multiple-choice college-admissions test. These problems had unique correct answers. While these provided a good starting point for our research, they were not well suited for collaborative knowledge building. Discourse around them was often confined to seeing who thought they knew the answer and then checking for correctness. For the VMT Spring Fests in 2005, 2006 and 2007, we moved to more involved math topics that could inspire several hours of joint inquiry.

Even with straightforward geometry problems, it became clear that students needed the ability to create, share and modify drawings within the VMT environment. We determined that we needed an object-oriented draw program, where geometric objects could be manipulated (unlike a pixel-based paint program). We contracted with the developers of ConcertChat to use and extend their text chat and shared whiteboard system, which is now available in Open Source. This system included a graphical referencing tool as well as social awareness and history features (Mühlpfordt \& Stahl, 2007). In order to help students find desirable chat rooms and to preserve team findings for all to see, we developed the VMT Lobby and integrated a Wiki with the Lobby and chat rooms (Stahl, 2008). Gradually, the technology and the math topics became much more complicated in response to the needs that were revealed when we analyzed the trials of the earlier versions of the VMT service. As the system matured, other research groups began to use it for their own trials, with their own math topics, procedures, analytic methods or even new technical 
features. These groups included researchers from Singapore, Rutgers, Hawai'i, Romania and Carnegie-Mellon (Stahl, 2009).

\section{The Nature Of the New Science}

The approach to chat interaction analysis that emerged in the VMT Project will now be discussed in terms of a number of issues (which correspond to general issues of most research methodologies, as indicated in parentheses):

\section{Group Cognition in a Virtual Math Team (Research Question)}

Learning-whether in a classroom, a workplace or a research lab-is not a simplistic memorization or storage of facts or propositions, as traditional folk theories had it. The term learning is a gloss for a broad range of phenomena, including: the development of tacit skills, the ability to see things differently, access to resources for problem solving, the discursive facility to articulate in a new vocabulary, the power to explain, being able to produce arguments or the making of new connections among prior understandings (Stahl \& Herrmann, 1999). We can distinguish these phenomena as taking place within individual minds, small-group interactions or communities of practice. The analysis of learning phenomena at these various levels of analysis requires different research methodologies, appropriate to corresponding research questions. The VMT Project was intended to explore the phenomena of group cognition and accordingly pursued the research question:

How does learning take place in small groups, specifically in small groups of students discussing math in a text-based online environment? What are the distinctive mechanisms or processes that take place at the small-group level of description when the group is engaged in problem-solving or knowledge-building tasks?

While learning phenomena at the other levels of analysis are important and interact strongly with the group level, we have tried to isolate and make visible the small-group phenomena and to generate a corpus of data for which the analysis of the group-level interactions can be distinguished from the effects of the individual and community levels.

The methods used to gather and analyze one's data should be appropriate to one's research question. To support such research, one must generate and collect data that are adequate for the selected kinds of analysis. Because we were interested in the group processes that take place in virtual math teams, we had to form teams that could meet together online. In the Spring Fests, students had to be able to come back together in the same teams on several subsequent occasions. The VMT environment had to be instrumented to record all messages and activities that were visible to the whole team in a way that could be played back by the analysts. The math problems and the feedback to the teams had to be designed to encourage the kinds of math discussions that would demonstrate processes of group cognition, such as formulating questions and proposals, coordinating drawings and textual narratives, checking proposed symbolic solutions, reviewing the team's work and so on. A sense of these desirable group activities and the skill of designing problems to encourage them had to develop gradually through the designbased research iterations. 


\section{Non-laboratory Experimental Design (Validity)}

Of course, to isolate the small-group phenomena we do not literally isolate our subject groups from individuals and communities. The groups consist of students, who are individuals and who make individual contributions to the group discourse based on their individual readings of the discourse. In addition, the groups exist and operate within community and social contexts, drawing upon the language and practices of their math courses and of their teen and online subcultures. These are essential features of a real-world context and we would not wish to exclude them even to the extent possible by confining the interaction to a controlled laboratory setting. We want the students to feel that they are in a natural setting, interacting with peers. We do not try to restrict their use of language in any way (e.g., by providing standardized prompts for chat postings or scripting their interactions with each other).

We are designing a service that can be used by students and others under a broad array of scenarios: integrated with school class work, as extra-curricular activities, as social experiences for home-schooled students, as cross-national team adventures or simply as opportunities (in a largely math-phobic world) to discuss mathematics. To get a sense of how such activities might work, we have to explore interactions in naturalistic settings, where the students feel like they are engaged in such activities rather than being laboratory subjects.

\section{Data Collection at the Group Level of Description (Unit of Analysis)}

Take the network of references in a chat-threading diagram (see Figure 1) as an image of meaning making at the group level (Stahl, 2007). One could almost say that the figure consists entirely of contributions from individuals (the chat postings and whiteboard drawings) and resources from the math community; that everything exists on either the individual or community level, not on the group level. Yet, what is important in the figure is the network of densely interwoven references, more than the objects that are connected by them. This network exists at the group level. It mediates the individual and the community by forming the joint problem space (Sarmiento, 2007; Teasley \& Roschelle, 1993), indexical ground (Hanks, 1992), referential network (Heidegger, 1927/1996) or situation (Suchman, 2007) within which meanings, significant objects and temporal relations are intersubjectively co-constructed (Dourish, 2001). On the individual level, these shared group meanings are interpreted and influence the articulation of subsequent postings and actions. On the community level, the meanings may contribute to a continually evolving culture through structuration processes (Giddens, 1984b). The VMT Project is oriented toward the processes at the group unit of analysis, which build upon, connect and mediate the individual and community phenomena. 


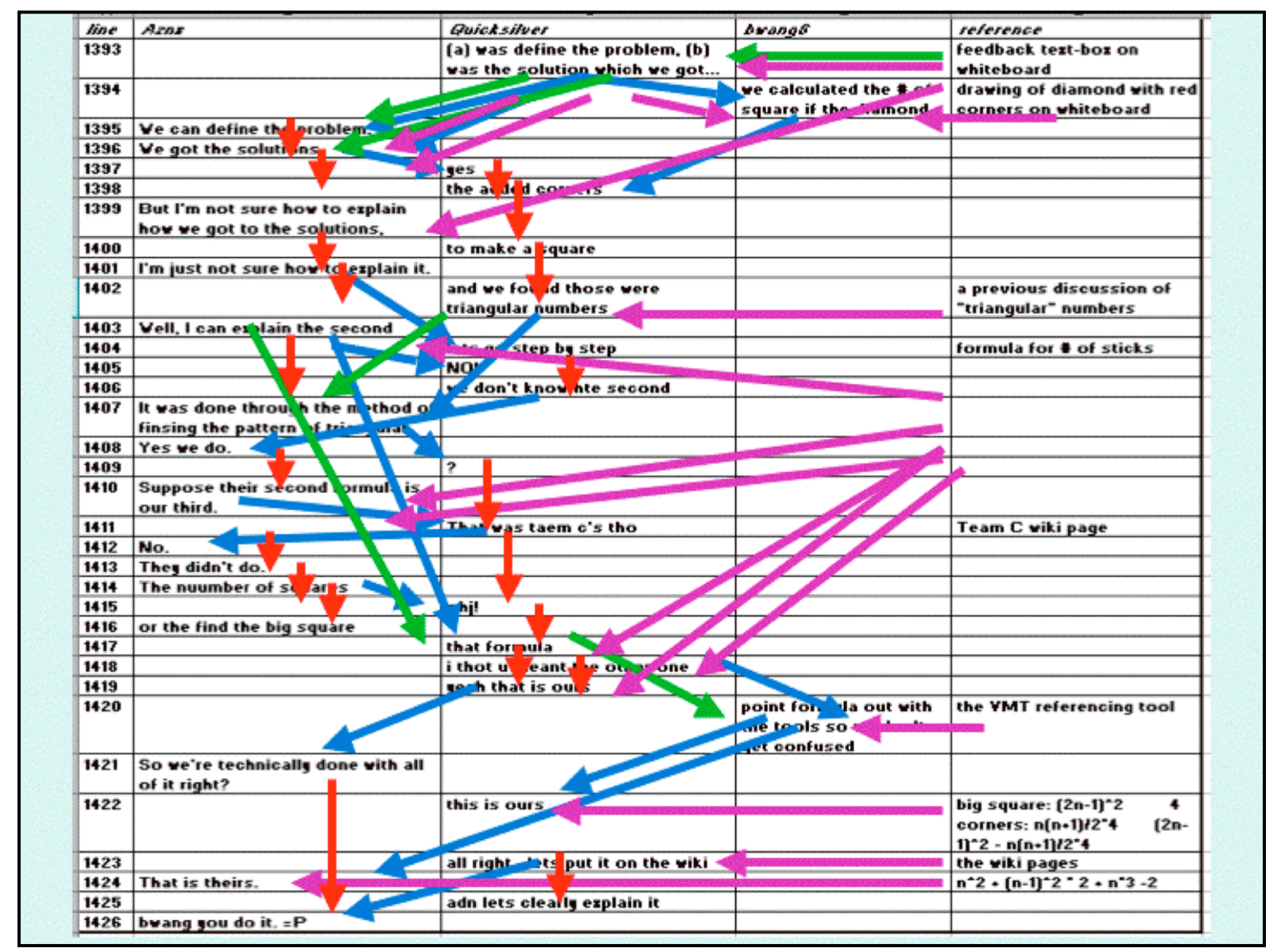

Figure 1. The network of references in a chat log excerpt.

Elements from the individual and community levels only affect the group level if they are referenced in the team's interaction. Therefore, we do not need to gather data about the students or their communities other than what appears in the interaction record. We do not engage in surveys or interviews of the students or their teachers. For one thing, the design of the VMT Project prohibits access to these sources of data, because the students are only available during the chat sessions. External sources of data would be of great interest for other research questions having to do with individual learning or cultural changes, but for our research question, they are unnecessary and might even form a distraction or skew our analysis because it would cause our readings of the postings to be influenced by information that the group had not had.

By moving to the disembodied online realm of group cognition in virtual math teams, it is easier for us to abandon the positivist metaphors of the mechanistic worldview. Not only is it clear that the virtual group does not exist in the form of a physical object with a persistent memory akin to a computer storage unit, but even the individual participants lack physical presence. All that exists when we observe the replayed chats are the traces of a discourse that took place years ago. Metaphors that might come naturally to an observer of live teamwork in a workplace or classroom-personalities, the group, learning, etc.- no longer seem fundamental. What exist immediately are the textual, graphical and symbolic inscriptions. These are significant fragments, whose meaning derives from the multi-layered references to each other 
and to the events, artifacts and agents of concern in the group discourse. This meaning is as fresh now as when the discourse originated, and can still be read off the traces by an analyst, much as by the original participants. This shows that the meanings shared by the groups are not dependent upon mental states of the individual students-although the students may have had interpretations of those meanings in mind, external to the shared experience. The form of our data reinforces our focus on the level of the shared-group-meaning making as an interactional phenomenon rather than a psychological one.

\section{Instrumentation and Data Formats (Objectivity)}

It was noted above that when one videotapes small-group interactions a number of practical problems arise. Data on face-to-face classroom collaboration runs into issues of (i) recording and transcribing the verbal interaction, (ii) capturing the visual interaction and (iii) knowing about all the influences on the interaction. The data is in effect already partially interpreted by selective placement of the microphone and camera. It is further interpreted by transcription of the talk, and is restricted by limited access to facial expressions and bodily gestures. Much happens in a classroom influencing the student teams that is not recorded.

The online setting of the VMT sessions eliminates many of these problems. As already described, the automatic computer log of the session captures everything that influences the group as a whole. This includes all the postings and whiteboard activity, along with their precise timing. They are captured at the same granularity as they are presented to the students. Chat postings appear as complete messages, defined by the author pressing the Enter button. Whiteboard textboxes appear as complete, when the author clicks outside of the textbox. Whiteboard graphics appear gradually, as each graphical element is positioned by the author. Computer-generated social-awareness messages (when people enter or exit the chat room, begin or end typing, move a graphical object, etc.) are also accurately recorded. The precision of the $\log$ recording is assured because it consists of the original actions (as implemented by the computer software) with their timestamps. The original display to the students is generated from the server using the same log data that is used by the VMT Replayer. There is no selectivity or interpretation imposed by the analysts in the preparation of the full session record.

For our analysis of chats, we use a VMT Replayer. The Replayer is simply an extended version of the Java applet that serves as the chat/whiteboard room in the VMT environment. The reproduced chat room is separated by a thin line at the bottom from a VCR-like interface for replaying the session (see Figure 2). The session can be replayed in real time or at any integral multiple of this speed. It can be started and stopped at any point. An analyst can drag the pointer along the timeline to scroll both the whiteboard history and the chat history in coordination. One can also step through the recorded actions, including all the awareness messages. In addition, spreadsheet logs can be automatically generated in various useful formats.

The data analyzed in the VMT Project is recorded with complete objectivity. There is no selectivity involved in the data generation, recording or collecting process. Furthermore, the complete recording can be made available to other researchers as a basis for their reviews of our analyses or the conducting of their own analyses. For instance, there have been multiple published analyses of the VMT data by other research groups following somewhat different research questions, theories and methods (Koschmann \& Stahl, 2009; Stahl, 2009). While collaborative sessions are each unique and in principle impossible to reproduce, it is quite 


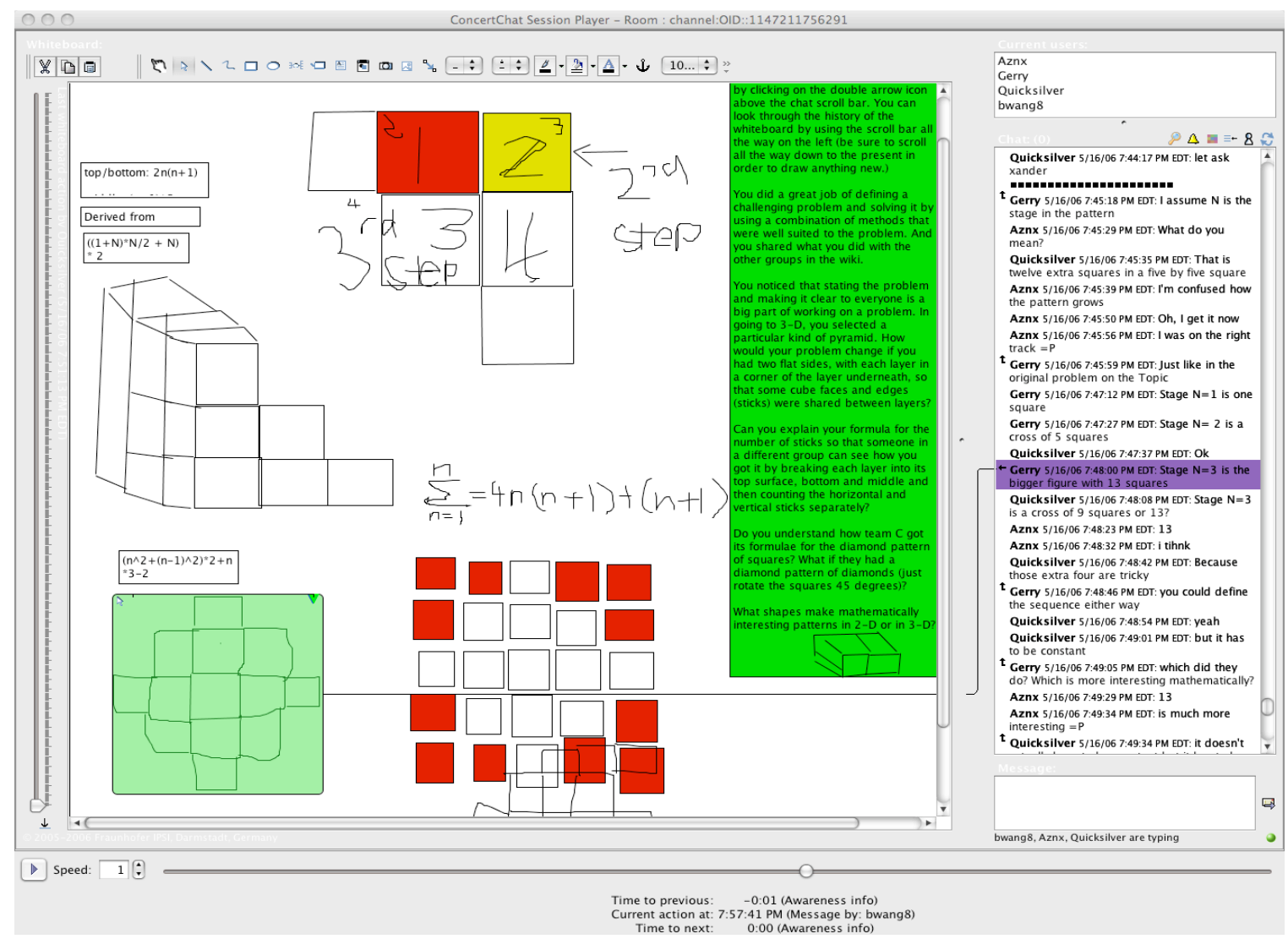

Figure 2. The VMT Replayer.

possible to reproduce the unfolding of a given session from the persistent, comprehensive and replayable record.

\section{Collaborative Data Sessions (Reliability)}

Interpretation of data in the VMT Project first begins with an attempt to describe what is happening in a chat session. We usually start this process with a data session (Jordan \& Henderson, 1995) involving six to twelve researchers. A typical data session is initiated by a researcher who is interested in having a particular segment of a session log discussed by the group. Generally, the segment seems to be both confusing and interesting in terms of a particular research question.

For our data sessions, we sit around a circle of tables and project an image of the VMT Replayer onto a screen visible to everyone. Most of us have laptop computers displaying the same Replayer, so that we can scan back and forth in the segment privately to explore details of the interaction that we may want to bring to the attention of the group. The group might start by playing the segment once or twice in real time to get a feel for how it unfolds. Then we typically go back to the beginning and discuss each line of the chat sequentially in some detail.

The interpretation of a given chat line becomes a deeply collaborative process. Generally, one person will make a first stab at proposing a hypothesis about the interactional work that line 
is doing in the logged discourse. Others will respond with suggested refinements or alternatives to the proposal. The group may then engage in exploration of the timing of chat posts, references back to previous postings or events, etc. Eventually the data analysis will move on to consider how the student group took up the posting. An interesting interpretation may require the analysts to return to earlier ground and revise their tentative previous understandings (Stahl, 2009, ch. $10)$.

The boundaries of a segment must be considered as an important part of the analysis. When does the interaction of interest really get started and when is it resolved? Often, increasingly deep analysis drives the starting point back as we realize that earlier occurrences were relevant.

It is usually first necessary to clarify the referential structure of the chat postings and how they relate to events in the whiteboard or to the comings and goings of participants. The threading of the chat postings provides the primary structure of the online, text-based discourse in much the same way that turn taking provides the core structure of spoken informal conversation. Because of the overlap in the typing of chat postings, it is sometimes tricky to figure out who is responding to what. Looking at the timestamps of posts and even at the timestamps of awareness messages about who is typing can provide evidence about what was visible when a posting was being typed. This can often suggest that a given post could or could not have been responding to a specific other post, although this is sometimes impossible to determine. When it is hard for the analyst to know the threading, it may have also been hard for most of the chat participants (other than the typist) to know; this may result in signs of trouble or misunderstandings in the subsequent chat.

The test of correctness of chat interaction analysis is not a matter of what was in individuals' minds, but of how postings function in the interaction. Most of the multi-layered referencing takes place without conscious awareness by the participants, who are experts at semantic, syntactic and pragmatic referencing and can design utterances in response to local resources without formulating explicit plans (Suchman, 2007). Thus, inspection of participants' memories would not reveal causes. Of course, participants could retroactively tell stories about why they posted what they did, but these stories would be based upon their current (not original) interpretations using their linguistic competence and upon their response to their current (not original) situation, including their sense of what the person interviewing them wants to hear. Thus, interpretations by the participants are not in principle privileged over those of the analyst and others with the relevant interpretive competence (Gadamer, 1960/1988). The conscious memories that a participant may have of the interaction are, according to Vygotsky's theory, just more interaction-but this time sub-vocal self-talk; if they were brought into the analysis, they would be in need of interpretation just as much as the original discourse.

Since our research question involves the group as the unit of analysis, we do not raise questions in the data session about what one student or another may have been doing, thinking or feeling as an individual. Rather, we ask what a given posting is doing interactionally within the group process, how it responds to and takes up other posts and what opportunities it opens for future posts. We look at how a post is situated in the sequential structure of the group discourse, in the evolving social order and in the team's meaning making. What is this posting doing here and now in the referential network? Why is it "designed to be read" (Livingston, 1995) in just this way? How else could it have been phrased and why would that not have achieved the same effect in the group discourse? 
We also look at how a given posting positions (Harré \& Moghaddam, 2003) both the author and the readers in certain ways. We do not attribute constant personalities or fixed roles to the individuals, but rather look at how the group is organized through the details of the discourse. Perhaps directing a question toward another student will temporarily bestow upon her a form of situated expertise (Zhou, Zemel \& Stahl, 2008) such that she is expected to provide an extended sequence of expository postings (Mercer \& Wegerif, 1999).

The discussion during a data session can be quite unorderly. Different people see different possible understandings of the log and propose alternative analyses. Generally, discussion of a particular posting continues until a consensus is tentatively established or someone agrees to look into the matter further and come back next week with an analysis. Notes are often taken on the data session's findings, but the productive result of the discussion most often occurs when one researcher is inspired to write about it in a conference paper or dissertation section. When ideas are taken up this way, the author will usually bring the more developed analysis back for a subsequent data session and circulate the paper.

In coding analysis, it is conventional to train two people to code some of the same log units and to compare their results to produce an inter-rater reliability measure (Strijbos \& Stahl, 2007). In our chat interaction analysis, we do not pretend that the $\log$ can be unproblematically partitioned into distinct units, which can be uniquely assigned to a small number of unambiguous codes. Rather, most interesting group discourse segments have a complex network of interwoven references. The analysis of such log segments requires a sophisticated human understanding of semantics, interpersonal dynamics, mathematics, argumentation and so on. Much is ultimately ambiguous and can be comprehended in multiple ways-sometimes the chat participants were intentionally ambiguous. At the same time, it is quite possible for analysts to make mistakes and to propose analyses that can be shown to be in error. To attain a reasonable level of reliability of our analyses, we make heavy use of data sessions. This ensures that a number of experienced researchers agree on the analyses that emerge from the data sessions. In addition, we try to provide logs - or even the entire session data with the Replayer-in our papers so that readers of our analyses can judge for themselves the interpretations that are necessarily part of chat analysis.

\section{Describing Social Practices (Generalizability)}

The research question that drives the VMT Project is: What are the distinctive mechanisms or processes that take place at the small-group level of description when the group is engaged in problem-solving or knowledge-building tasks? Therefore, we are interested in describing the inter-personal practices of the groups that interact in the VMT environment. There are, of course, many models and theories in the learning sciences describing the psychological practices of individuals involved in learning. At the opposite extreme, Lave \& Wenger's (1991) theory of situated learning describes social practices of communities of practice, whereby a community renews itself by moving newcomers into increasingly central forms of legitimate peripheral participation. However, there are few descriptions specifically of how small groups engage in learning practices.

Vygotsky (1930/1978) argued that learning takes place inter-subjectively (in dyads or groups) before it takes place intra-subjectively (by individuals). For instance, in his analysis of the infant and mother (p. 56), he outlines the process through which an infant's unsuccessful grasping at some object becomes established by the mother-child dyad as a pointing at the object. 
This shared practice of pointing subsequently becomes ritualized by the dyad (LeBaron \& Streeck, 2000) and then mediated and "internalized" by the infant as a pointing gesture. The pointing gesture - as a foundational form of deictic reference - is a skill of the young child, which he can use for selecting objects in his world and learning about them. The gesture is understood by his mother because it was intersubjectively established with her. In this prototypical example, Vygotsky describes learning as an inter-subjective or small-group practice of a dyad.

While we can imagine that Vygotsky's description is based on a concrete interaction of a specific infant and mother in a particular time and place, the pointing gesture that he analyzed is ubiquitous in human culture. In this sense, the analysis of a unique interaction can provide a generalizable finding. The science of ethnomethodology (the study of the methods used by people) (Garfinkel, 1967) is based on the fact that people in a given culture or linguistic community share a vast repertoire of social practices for accomplishing their mundane tasks. It is only because we share and understand this stock of practices that we can so quickly interpret each other's verbal and gestural actions, even in novel variations under unfamiliar circumstances. The analysis of unique case studies can result in the description of social practices that are generalizable (Maxwell, 2004). The methods developed in specific situated encounters are likely to be typical of a broad range of cases under similar conditions.

In our data sessions, we find the same kinds of moves occurring in case after case that we analyze. On the one hand, group methods are extremely sensitive to changes in the environment, such as differences in features and affordances of the communication media. On the other hand, groups of people tend to adapt widespread methods of interaction to changing circumstances in similar ways - to support general human and social needs. Group methods are not arbitrary, but draw on rich cultural stocks of shared behavior and adapt the outward appearances in order to maintain the underlying structure under different conditions.

By describing the structure of group methods in detailed case studies, we can characterize general methods of group behavior, group learning or group cognition. Findings from analyses of case studies can lead to the proposal of theoretical categories, conceptualizations, structures or principles - in short, to a science of group interaction.

\section{The Foundational Role of Group Cognition}

As discussed above, students in virtual math teams are active as individuals, as group participants and as community members. They each engage in their own, private individual activities, such as reading, interpreting, reflecting upon and typing chat messages. Their typed messages also function as group actions, contributing to the on-going problem solving of the team. Viewed as community events, the chats participate in the socialization process of the society, through which the students become increasingly skilled members of the community of mathematically literate citizens.

A thesis of the theory of group cognition is, "Small groups are the engines of knowledge building. The knowing that groups build up in manifold forms is what becomes internalized by their members as individual learning and externalized in their communities as certifiable knowledge" (Stahl, 2006, p. 16). Despite their centrality, small groups have not been theorized or studied extensively. 
Some small-group literature has been produced from either the methodological perspective of psychology or that of sociology, primarily since World War II. Traumatized by the massculture horrors of fascism and by extreme forms of mentalist pseudo-science, these predominantly behaviorist studies focused on the negative aspects of "group think" and caricatured the notion of "group mind"- which had a well-respected history before the rise of positivism (Wegner, 1986). These studies miss the pivotal role of small groups in processes of learning.

More recent theories like distributed cognition, situated action or activity theory actually conduct case studies of small-group interaction, but they do not theorize the small group as their unit of analysis and therefore they do not produce descriptions of small-group methods as such. Even Hutchins (1996)), in studying distributed cognition in the wild, does not thematize the interpersonal interactions, but focuses on the cognitive unit of analysis, simply broadening it to include the external computational and physical representational artifacts that an individual worker uses. Furthermore, the cognitive accomplishments he studies are fundamentally routine, well scripted procedures that do not involve creative solutions to ill-structured problems; the coordination of the navigational team is fixed by naval protocol, not co-constructed through the interaction, although it must still be enacted in concrete situations.

The VMT studies provide a model for describing the small-group methods as distinct from individual behaviors and community practices. They look at rich interactions in groups larger than dyads, where individual identities play a smaller role. They analyze group efforts in highorder cognition such as mathematical problem solving and reflection on the group problemsolving trajectory. They investigate groups that meet exclusively online, where the familiar visual, physical and aural modes of communication are unavailable, and where communication is mediated by designed technological environments.

Understanding how a collaborative group as a whole constructs knowledge through joint activity in a CSCL setting is what sets the science of group cognition apart from other approaches to the study of learning. Successful collaboration involves not only the incorporation of contributions of individuals into the group discourse, but also the effort to make sure that participating individuals understand what is taking place at the group level. The contributions of individuals to the group and of understandings from the group to the individuals cannot be studied by analyses at the individual unit of analysis, but only be studying the interactions at the group level. The group knowledge-construction process synthesizes innumerable resources from language, culture, the group's own history, individual backgrounds, relevant contexts and the sequential unfolding of the group discourse in which the individuals participate. Although the group process is dependent upon contributions and understanding of individuals, their individual cognition is essentially situated in the group process. Group cognition is the science of cognitive processes at the group unit of analysis. These group processes - such as the sequential flow of proposals, questioning, building common ground, maintaining a joint problem space, establishing intersubjective meanings, positioning actors in evolving roles, building knowledge collaboratively and solving problems together - are not analyzable as individual behaviors.

There is a scientific lacuna within the learning sciences between sciences of the individual and sciences of communities. There are important cognitive achievements at the small-group level of description, which should be studied by a science of groups. Online small groups are becoming increasingly possible and important in the global networked world, and a post- 
cognitive science of virtual groups could help the design of collaborative software for working and learning. It could provide an effective foundation for the new science of learning.

\section{REFERENCES}

Adams, F., \& Aizawa, K. (2008). The bounds of cognition. Malden, MA: Blackwell.

Barron, B. (2000). Achieving coordination in collaborative problem-solving groups. Journal of The Learning Sciences, 9(4), 403-436.

Barron, B. (2003). When smart groups fail. The Journal of the Learning Sciences, 12(3), 307-359.

Bereiter, C. (2002). Education and mind in the knowledge age. Hillsdale, NJ: Lawrence Erlbaum Associates.

Bourdieu, P. (1972/1995). Outline of a theory of practice (R. Nice, Trans.). Cambridge, UK: Cambridge University Press.

Çakır, M. P., Zemel, A., \& Stahl, G. (2009). The joint organization of interaction within a multimodal CSCL medium. International Journal of Computer-Supported Collaborative Learning, 4(2), 115149. Available at http://dx.doi.org/10.1007/s11412-009-9061-0.

Clinton, H. (1996). It takes a village: And other lessons children teach us. New York, NY: Simon \& Shuster.

Cohen, E. G., Lotan, R. A., Abram, P. L., Scarloss, B. A., \& Schultz, S. E. (2002). Can groups learn? Teachers College Record, 104(6), 1045-1068.

Dourish, P. (2001). Where the action is: The foundations of embodied interaction. Cambridge, MA: MIT Press.

Dreyfus, H. (1972). What computers cannot do. New York, NY: Harper and Row.

Engeström, Y. (1987). Learning by expanding: An activity-theoretical approach to developmental research. Helsinki, Finland: Orienta-Kosultit Oy.

Engeström, Y. (2008). From teams to knots. Cambridge, UK: Cambridge University Press.

Gadamer, H.-G. (1960/1988). Truth and method. New York, NY: Crossroads.

Garfinkel, H. (1967). Studies in ethnomethodology. Englewood Cliffs, NJ: Prentice-Hall.

Geertz, C. (1973). The interpretation of cultures. New York, NY: Basic Books.

Giddens, A. (1984a). Elements of the theory of structuration. In The constitution of society (pp. 1-40): U of California Press.

Giddens, A. (1984b). The constitution of society. Outline of the theory of structuration. Berkeley, CA: U of California Press.

Hanks, W. (1992). The indexical ground of deictic reference. In C. Goodwin \& A. Duranti (Eds.), Rethinking context: Language as an interactive phenomenon. Cambridge, UK: Cambridge University Press.

Harré, R., \& Moghaddam, F. (2003). Introduction: The self and others in traditional psychology and in positioning theory. In R. Harré \& F. Moghaddam (Eds.), The self and others (pp. 1- 11). Westport, CT: Praeger.

Hegel, G. W. F. (1807/1967). Phenomenology of spirit (J. B. Baillie, Trans.). New York, NY: Harper \& Row.

Heidegger, M. (1927/1996). Being and time: A translation of Sein und Zeit (J. Stambaugh, Trans.). Albany, NY: SUNY Press.

Hollan, J., Hutchins, E., \& Kirsh, D. (2000). Distributed cognition: Toward a new foundation of humancomputer interaction research. ACM Transactions on Computer-Human Interaction, 7(2), 174-196.

Husserl, E. (1917/1991). On the phenomenology of internal time (1893-1917) (J. Brough, Trans.). Dodrecht, NL: Kluwer.

Hutchins, E. (1996). Cognition in the wild. Cambridge, MA: MIT Press. 
Johnson, D. W., \& Johnson, R. T. (1989). Cooperation and competition: Theory and research. Edina, $\mathrm{MN}$ : Interaction Book Company.

Jordan, B., \& Henderson, A. (1995). Interaction analysis: Foundations and practice. Journal of the Learning Sciences, 4(1), 39-103. Available at http://lrs.ed.uiuc.edu/students/cmerkel/document4.HTM.

Koschmann, T. (1996). Paradigm shifts and instructional technology. In T. Koschmann (Ed.), CSCL: Theory and practice of an emerging paradigm (pp. 1-23). Mahwah, NJ: Lawrence Erlbaum.

Koschmann, T., \& Stahl, G. (2009 of Conference). Symposium: Examining practices of computermediated learning. Paper presented at the international conference on Computer Support for Collaborative Learning (CSCL 2009), Rhodes, Greece. Available at http://GerryStahl.net/pub/cscl2009koschmann.pdf.

Latour, B. (2007). Reassembling the social: An introduction to actor-network-theory. Cambridge, UK: Cambridge University Press.

Lave, J., \& Wenger, E. (1991). Situated learning: Legitimate peripheral participation. Cambridge, UK: Cambridge University Press.

LeBaron, C., \& Streeck, J. (2000). Gesture, knowledge and the world. In D. McNeill (Ed.), Language and gesture (pp. 118-138). Cambridge, UK: Cambridge University Press.

Lerner, G. (1993). Collectivities in action: Establishing the relevance of conjoined participation in conversation. Text, 13(2), 213-245.

Livingston, E. (1987). Making sense of ethnomethodology. London, UK: Routledge \& Kegan Paul.

Livingston, E. (1995). An anthropology of reading. Bloomington: IN: Indiana University Press.

Marx, K. (1867/1976). Capital (B. Fowkes, Trans. Vol. I). New York, NY: Vintage.

Maxwell, J. (2004). Causal explanation, qualitative research, and scientific inquiry in education. Educational Researcher, 33(2), 3-11. Available at http://www.aera.net/pubs/er/pdf/vol33 02/202602 pp03-11.pdf.

Mercer, N., \& Wegerif, R. (1999). Is "Exploratory talk" Productive talk? In K. Littleton \& P. Light (Eds.), Learning with computers: Analyzing productive interaction (pp. 79-101). New York, NY: Routledge.

Merleau-Ponty, M. (1945/2002). The phenomenology of perception (C. Smith, Trans. 2 ed.). New York, NY: Routledge.

Mühlpfordt, M., \& Stahl, G. (2007). The integration of synchronous communication across dual interaction spaces. In C. Chinn, G. Erkens \& S. Puntambekar (Eds.), The proceedings of CSCL 2007: Of mice, minds, and society (CSCL 2007). New Brunswick, NJ. Available at http://GerryStahl.net/vmtwiki/martin.pdf.

Piaget, J. (1985). The equilibrium of cognitive structures: The central problem of intellectual development: Chicago University Press.

Polanyi, M. (1962). Personal knowledge. London, UK: Routledge \& Kegan Paul.

Robbins, P., \& Aydede, M. (Eds.). (2009). The Cambridge handbook of situated cognition. Cambridge, UK: Cambridge University Press.

Sacks, H. (1962/1995). Lectures on conversation. Oxford, UK: Blackwell.

Sarmiento, J. (2007). Bridging: Interactional mechanisms used by online groups to sustain knowledge building over time. Paper presented at the international conference on Computer-Supported Collaborative Learning (CSCL '07), New Brunswick, NJ. Available at http://GerryStahl.net/vmtwiki/johann.pdf.

Sawyer, R. K. (2003). Group creativity: Music, theater, collaboration. Mahwah, NJ: Lawrence Erlbaum.

Scardamalia, M., \& Bereiter, C. (1996). Computer support for knowledge-building communities. In T. Koschmann (Ed.), CSCL: Theory and practice of an emerging paradigm (pp. 249-268). Hillsdale, NJ: Lawrence Erlbaum Associates.

Schegloff, E. A. (2007). Sequence organization in interaction: A primer in conversation analysis. Cambridge, UK: Cambridge University Press.

Schön, D. A. (1983). The reflective practitioner: How professionals think in action. New York, NY: Basic Books. 
Schwartz, D. (1995). The emergence of abstract representations in dyad problem solving. Journal of the Learning Sciences, 4(3), 321-354.

Stahl, G. (2002). Rediscovering CSCL. In T. Koschmann, R. Hall \& N. Miyake (Eds.), CSCL 2: Carrying forward the conversation (pp. 169-181). Hillsdale, NJ: Lawrence Erlbaum Associates. Available at http://GerryStahl.net/cscl/papers/ch01.pdf.

Stahl, G. (2004a). Mediation of group cognition. SigGroup Bulletin, 24(4), 13-17. Available at http://GerryStahl.net/publications/journals/Mediation\%20of\%20Group\%20Cognition.pdf.

Stahl, G. (2004b). Thinking at the group unit of analysis. Paper presented at the CSCL SIG Symposium of the European Union Kaleidoscope Network of Excellence (KAL '04), Lausanne, Switzerland. Available at http://GerryStahl.net/pub/kal2004.pdf \& http://GerryStahl.net/pub/kal2004ppt.pdf.

Stahl, G. (2005). Group cognition: The collaborative locus of agency in CSCL. Paper presented at the international conference on Computer Support for Collaborative Learning (CSCL '05), Taipei, Taiwan. Proceedings pp. 632-640: Lawrence Erlbaum Associates. Available at http://GerryStahl.net/pub/cscl2005.pdf \& http://GerryStahl.net/pub/cscl2005ppt.pdf.

Stahl, G. (2006). Group cognition: Computer support for building collaborative knowledge. Cambridge, MA: MIT Press. Available at http://GerryStahl.net/mit/.

Stahl, G. (2007). Meaning making in CSCL: Conditions and preconditions for cognitive processes by groups. Paper presented at the international conference on Computer-Supported Collaborative Learning (CSCL '07), New Brunswick, NJ: ISLS. Available at http://GerryStahl.net/pub/csc107.pdf.

Stahl, G. (2008). Integrating a wiki into support for group cognition. Paper presented at the International Conference of the Learning Sciences (ICLS 2008), Utrecht, Netherlands. Available at http://GerryStahl.net/pub/icls2008wiki.pdf.

Stahl, G. (2009). Studying virtual math teams. New York, NY: Springer. Available at http://GerryStahl.net/vmt/book.

Stahl, G., \& Herrmann, T. (1999). Intertwining perspectives and negotiation. Paper presented at the ACM SIGGROUP Conference on Supporting Group Work (Group '99), Phoenix, AZ. Proceedings pp. 316324. Available at http://GerryStahl.net/cscl/papers/ch07.pdf.

Strijbos, J. W., \& Stahl, G. (2007). Methodological issues in developing a multi-dimensional coding procedure for small group chat communication. Learning \& Instruction. Special issue on measurement challenges in collaborative learning research, 17(4), 394-404. Available at http://GerryStahl.net/vmtwiki/jw.pdf.

Suchman, L. (1987). Plans and situated actions: The problem of human-machine communication. Cambridge, UK: Cambridge University Press.

Suchman, L. A. (2007). Human-machine reconfigurations: Plans and situated actions (2nd ed.). Cambridge, UK: Cambridge University Press.

Teasley, S. D., \& Roschelle, J. (1993). Constructing a joint problem space: The computer as a tool for sharing knowledge. In S. P. Lajoie \& S. J. Derry (Eds.), Computers as cognitive tools (pp. 229-258). Mahwah, NJ: Lawrence Erlbaum Associates, Inc.

Vygotsky, L. (1930/1978). Mind in society. Cambridge, MA: Harvard University Press.

Wegner, D. (1986). Transactive memory: A contemporary analysis of the group mind. In B. Mullen \& G. R. Goethals (Eds.), Theories of group behavior (pp. 185-208). New York, NY: Springer Verlag.

Winograd, T., \& Flores, F. (1986). Understanding computers and cognition: A new foundation of design. Reading, MA: Addison-Wesley.

Wittgenstein, L. (1953). Philosophical investigations. New York, NY: Macmillan.

Zemel, A., \& Çakir, M. P. (2007). Reading's work: The mechanisms of online chat as social interaction. Paper presented at the National Communication Association Convention, Chicago, IL. Available at http://GerryStahl.net/vmtwiki/alan2.pdf.

Zhou, N., Zemel, A., \& Stahl, G. (2008). Questioning and responding in online small groups engaged in collaborative math problem solving. Paper presented at the International Conference of the Learning Sciences (ICLS 2008), Utrecht, Netherlands. Available at http://GerryStahl.net/pub/icls2008nan.pdf. 
